\title{
HBx co-localizes with COXIII in HL-7702 cells to upregulate mitochondrial function and ROS generation
}

\author{
LAI-YU ZOU ${ }^{1}$, BI-YUN ZHENG ${ }^{2}$, XUE-FEN FANG ${ }^{2}$, DAN LI $^{2}$, YUE-HONG HUANG ${ }^{2}$, \\ ZHI-XIN CHEN ${ }^{2}$, LIN-YING ZHOU ${ }^{3}$ and XIAO-ZHONG WANG ${ }^{2}$ \\ Departments of ${ }^{1}$ Infection and ${ }^{2}$ Gastroenterology, Union Hospital of Fujian Medical University; \\ ${ }^{3}$ Laboratory of Electron Microscopy, Fujian Medical University, Gulou, Fuzhou, Fujian 350001, P.R. China \\ Received November 16, 2014; Accepted February 19, 2015
}

DOI: $10.3892 /$ or.2015.3852

\begin{abstract}
Hepatocellular carcinoma (HCC) is one of the most common malignant diseases, and $\mathrm{HBx}$ leads to the development of HBV-associated HCC. Mitochondria are key organelles that regulate apoptosis, cellular energetics and signal transduction pathways, and are the source of $\mathrm{HBx}$-induced reactive oxygen species (ROS). Recent findings have shown that HBx interacts with the inner mitochondrial membrane protein, COXIII, via the yeast two-hybrid system, mating experiment and coimmunoprecipitation. The aim of the present study was to examine the co-localizaiton of HBx and COXIII in HL-7702 cells and to investigate ensuing alterations of mitochondrial function. An HL-7702 cell line stably expressing the $H B x$ gene by lentivirus vectors was constructed. Confocal microscopy was utilized to assess the interaction between $\mathrm{HBx}$ protein and COXIII. Expression of COXIII, activities of cytochrome $c$ oxidase $(\mathrm{COX})$ and the mitochondrial membrane potential, which were functionally relevant to the HBx protein-COXIII interaction, were investigated in cell cultures. Moreover, the intracellular ROS levels were detected by flow cytometry. The results demonstrated that $\mathrm{HBx}$ co-localized with the inner mitochondrial protein, COXIII, in HL-7702 cells, causing the upregulation of COXIII protein expression as well as COX activity. However, HBx did not alter the mitochondrial membrane potential and mitochondria exhibited only slight swelling in HL-7702-HBx cells. Moreover, HBx elevated the generation of mitochondrial ROS in HL-7702-HBx cells. The main finding of the present study was that the co-localization of HBx and COXIII leads to upregulation of the mitochondrial function and ROS generation, which are associated with the oncogenesis of HBV-associated HCC.
\end{abstract}

Correspondence to: Professor Xiao-Zhong Wang, Department of Gastroenterology, Union Hospital of Fujian Medical University, 29 Xinquan Road, Gulou, Fuzhou, Fujian 350001, P.R. China E-mail: drwangxz@163.com

Key words: hepatitis $\mathrm{B}$ virus $\mathrm{X}$ protein, cytochrome $c$ oxidase subunit III, mitochondrial function, reactive oxygen species, hepatocellular carcinoma

\section{Introduction}

Hepatocellular carcinoma (HCC) remains one of the most prevalent malignant diseases and has the fourth highest mortality rate worldwide (1). Chronic $\mathrm{HBV}$ infection remains the major etiological factor of HCC worldwide with $>50 \%$ of HCC patients being chronic carriers (2). The activities of $\mathrm{HBx}$ are known to play major roles in the onset and progression of HBV-associated HCC. HBx, encoded by the smallest open reading frame of mammalian hepadnaviruses, is 154 amino acids in size, with a molecular mass of $\sim 17.5 \mathrm{kDa}(3)$. HBx protein interacts with different host factors to modulate cell signal transduction pathways, transcriptional regulations, cell cycle progression, DNA repair, apoptosis and genetic stability (1).

HBx is predominately localized in the cytoplasm with a low level of nuclear distribution (4). Mounting evidence from immunofluorescence microscopy and subcellular fractionation techniques have demonstrated that a fraction of cytosolic HBx co-localizes with mitochondria in primary rat hepatocytes (5), in the human WRL68 liver cell line and human HepG2 hepatocarcinoma cell line (6), in Huh7 (7) and in HBx-expressing COS cells, a monkey kidney cell line (8), and in HBx-transgenic mouse hepatocytes (9), suggesting a key role of $\mathrm{HBx}$ in affecting mitochondrial physiology, metabolism and other relevant functions. The abovementioned studies explain using different cell lines that $\mathrm{HBx}$ localizes to the outer mitochondrial membrane (10). However, our recent studies have shown that the inner mitochondrial membrane protein, COXIII, also interacts with hepatitis B virus X protein in vivo by the yeast two-hybrid system, further verified by mating experiment, coimmunoprecipitation and confocal laser scanning microscopy (11-13).

Mitochondria are key organelles that regulate apoptosis, cellular energetics and signal transduction pathways (14), and are the source of HBx-induced ROS (15). Accumulating evidence has suggested that inflammation contributes to HCC development due to the adverse effects of inflammatory mediators such as proinflammatory cytokine and reactive oxygen species (ROS). They play a key role on DNA repair, DNA methylation, DNA oxidation and lipid peroxidation $(16,17)$. There are five enzyme complexes (complexes I-V) involved in oxidative phosphorylation in mitochondria. Cytochrome $c$ 
oxidase (COX; or complex IV), composed of the COXI, COXII and COXIII mtDNA encoded subunits and 10 nuclear DNA encoded subunits, is an essential component of the respiratory chain that catalyzes the reduction of molecular oxygen by reduced cytochrome $c$ (18). COX is the terminal enzyme of the ETC and plays a pivotal role in the generation of ATP and maintenance of the mitochondrial transmembrane potential $(\Delta \Psi \mathrm{m})$. Increased expression of COX subunits is associated with restoration of COX activity, increased cellular ATP levels, and a delayed restoration of $\Delta \Psi \mathrm{m}$ (19). In human colorectal carcinoma cell lines metastasis is closely associated with COXIII, which functions in the process of growth and differentiation, transcription, apoptosis and signal transduction (20). Furthermore, upregulation of mitochondrial energy production-associated genes, $C O X I I I$ and $C O X V b$, are capable of enhancing cell growth by supporting higher energy requirements for cells $(21,22)$. The abovementioned findings indicate that changes of mitochondrial function may be important in the HBX-associated HCC.

In the present study, we used HL-7702 cells which expressed HBx stably as a biologically relevant system to explore the physiological effect of HBx. We found that HBx located to the inner mitochondrial membrane protein COXIII, leading to alterations of mitochondrial function and the subsequent upregulation of inflammatory mediator, ROS.

\section{Materials and methods}

Antibodies and reagents. Anti-DYKDDDDK-Tag (anti-flag) antibody was purchased from Abmart (Shanghai, China). Anti-cytochrome $c$ oxidase subunit III (anti-COXIII) (N-20) antibody was purchased from Santa Cruz Biotechnology, Inc. (Santa Cruz, CA, USA). Anti- $\beta$-actin antibody was purchased from ZSGB-BIO (Beijing, China). $\mathrm{CF}^{\mathrm{TM}} 488$-conjugated donkey anti-mouse $\operatorname{IgG}(\mathrm{H}+\mathrm{L}), \mathrm{CF}^{\mathrm{TM}} 350$-conjugated donkey anti-goat $\operatorname{IgG}(\mathrm{H}+\mathrm{L})$, and puromycin were obtained from Sigma (St. Louis, MO, USA). MitoTracker Red was obtained from Molecular Probes (Eugene, OR, USA). Primers were produced by Biosune (Shanghai, China).

Plasmids. The HBx expression plasmid PcDNA3.1-x (HBV subtype ayw) was a gift from Professor Michael J. Bouchard (Drexel University College of Medicine, Philadelphia, PA, USA). The lentivirus packaging system: pLOV.CMV.eGFP.2A. EF1a.PuroR (pLOV), psPAX2 and pMD2.G were provided by Neuron Biotech Co., Ltd. (Shanghai, China). The lentivirus vector pLOV contains the gene coding for green fluorescent protein (GFP), puromycin and the peptide DYKDDDDK (flag).

Cells cultures. The human 293T embryonic kidney cell line was provided by ATCC (UK). The human HL-7702 hepatocytes cell line was purchased from the Shanghai Cell Bank (Shanghai, China). The 293T and HL-7702 cells were grown in Dulbecco's modified Eagle's medium (DMEM) containing $10 \%$ fetal bovine serum (FBS) and 1\% penicillin-streptomycin solution and maintained at $37^{\circ} \mathrm{C}$ in a humidified atmosphere composed of $95 \%$ air and $5 \% \mathrm{CO}_{2}$.

Generation of the recombinant lentivirus and establishment of the stably transfected HL-7702 cell line. Full-length HBx
(465 bp) was amplified from PcDNA3.1-x by polymerase chain reaction (PCR) and then infused with flag epitope at the N-terminus and then cloned into pLOV. The plasmid was digested with $X b a$ I to construct the recombinant lentiviral vector pLOV.flag-HBx. The recombinant lentiviral particles were generated by transient cotransfection with $293 \mathrm{~T}$ cells by the three-plasmid expression system, and harvested by filtration through a $0.45 \mu \mathrm{m}$ filter and ultracentrifugation at $100,000 \mathrm{xg}$ for $2 \mathrm{~h}$ at $4^{\circ} \mathrm{C}$. The recombinant lentiviral particles titer was calculated by qPCR and used to transduce HL-7702 cells. The cell clones were then treated with $0.2 \mu \mathrm{g} / \mathrm{ml}$ puromycin for 10 days. Expression of GFP in the objective clones was examined directly by fluorescence microscopy. Positive clones expressing GFP and resistant to puromycin were screened and designated as HL-7702-HBx and HL-7702mock, respectively. $\mathrm{HBx}$, mock and control were considered to represent HL-7702-HBx, HL-7702-mock and HL-7702 cells, respectively. The expression of flag- $\mathrm{HBx}$ was detected by qPCR and western blot analysis.

Indirect immunofluorescence. To eliminate the impact of green fluorescence caused by GFP in HL-7702-HBx cells, we used $100 \%$ methanol as a fixative (23). As a result, green fluorescence disappeared (data not shown). The distributions of HBX and COXIII proteins were measured by confocal microscopy. The cells were seeded directly onto coverslips and incubated for $48 \mathrm{~h}$. When the density of cells achieved $60-70 \%$, the culture medium was removed by repeated washes with phosphate-buffered saline (PBS), and the cells were stained for 30 min with $150 \mathrm{nM}$ MitoTracker Red. The cells were first fixed with a $100 \%$ ice methanol solution, preincubated in blocking solution (5\% donkey serum albumin in PBS) and incubated with the appropriate primary antibodies (anti-flag and anti-COXIII) at $4^{\circ} \mathrm{C}$ overnight. After overnight incubation, the cells were washed. The fluorescence-labeled secondary antibodies $\left(\mathrm{CF}^{\mathrm{TM}} 488\right.$-conjugated donkey antimouse, $\mathrm{CF}^{\mathrm{TM}} 350$-conjugated donkey anti-goat) were added and incubated for $60 \mathrm{~min}$ at $37^{\circ} \mathrm{C}$ in the dark, and the sections were mounted using glycerol. Fluorescence images were captured by confocal laser scanning microscopy (Leica, Germany).

Isolation of mitochondria and measurement of COX activity. Mitochondria were isolated using the mitochondrial isolation kit for mammalian cells (Thermo Scientific, Waltham, MA, USA) according to the manufacturer's instructions. Briefly, following lysis of $\sim 2 \times 10^{7}$ cells, cell debris and nuclei were pelleted at $700 \times \mathrm{g}$ for $10 \mathrm{~min}$ at $4^{\circ} \mathrm{C}$, followed by centrifugation at $3,000 \times \mathrm{g}$ for $15 \mathrm{~min}$ at $4^{\circ} \mathrm{C}$ to pellet a mitochondrially enriched fraction, and then $12,000 \mathrm{x} \mathrm{g}$ for $5 \mathrm{~min}$ at $4^{\circ} \mathrm{C}$ to pellet the isolated mitochondria. The isolated mitochondria were vortexed with $30 \mu 1$ of $1 \mathrm{X}$ enzyme dilution buffer (10 mM Tris- $\mathrm{HCl}, \mathrm{pH} 7.0$, containing $250 \mathrm{mM}$ sucrose). Protein concentrations were measured using the bicinchoninic acid (BCA) assay and then adjusted to $1 \mu \mathrm{g} /$ $\mu 1$. COX activity was determined using the Cytochrome $c$ Oxidase Assay kit (GenMed, Shanghai, China) according to the manufacturer's instructions. COX activity was based on a colorimetric assay that quantifies the oxidation of ferrocytochrome $c$ to ferricytochrome $c$ via cytochrome $c$ oxidase, a reaction that results in a decrease in absorbance at $550 \mathrm{~nm}$. 
This decrease was monitored by an enzyme mark instrument (Bio-Tek, Winooski, VT, USA) calibrated to zero. Isolated mitochondria $(10 \mu \mathrm{l})$ were combined with $10 \mu \mathrm{l}$ of lysis buffer, then mixed with $205 \mu \mathrm{l}$ of buffer solution in 96-well plates. The reaction was initiated by the addition of $25 \mu \mathrm{l}$ of reaction liquid, and the decrease in absorbance at $550 \mathrm{~nm}$ was measured for $1 \mathrm{~min}$. Activity was calculated based on the equation: $\mathrm{U} / \mathrm{ml}=[(\Delta$ Abs550/min for the sample $-\Delta$ Abs550/min for the blank) $\mathrm{x}$ dilution factor $\mathrm{x}$ total reaction volume]/[mitochondria isolate volume $\mathrm{x}$ the difference in extinction coefficients between ferro- and ferri-cytochrome $c$ at $550 \mathrm{~nm}$ (21.84)]. One unit was considered the amount that oxidizes $1 \mu \mathrm{mol}$ reduced cytochrome $c / \mathrm{min}$ at $\mathrm{pH} 7.0$ and $25^{\circ} \mathrm{C}$.

Flow cytometric analysis. Cells in the three groups were harvested with trypsin and resuspended in PBS $\left(1 \times 10^{6}\right.$ cells $\left./ \mathrm{ml}\right)$ to analyze $\Delta \Psi \mathrm{m}, \mathrm{ROS}$ and cell cycles by flow cytometry. The $\Delta \Psi \mathrm{m}$ was assessed using the cationic fluorescent dye, TMRM. When cells reached $1 \times 10^{6}$ cells $/ \mathrm{ml}$, they were incubated in $100 \mathrm{nM}$ TMRM solution at $37^{\circ} \mathrm{C}$ in the dark for $20 \mathrm{~min}$. The cells were washed twice with PBS and resuspended in PBS for analysis by a flow cytometer. Intracellular ROS levels were subsequently detected by staining cells with $50 \mu \mathrm{M}$ dihydroethidium (DHE) fluorescence probe (Vigorous Biotechnology, Beijing, China) for $30 \mathrm{~min}$ in the dark. The cells were collected by trypsinization and the mean fluorescence intensity was quantified by flow cytometry.

Transmission electron microscopy (TEM). The morphological changes of cell and mitochondria were observed by TEM. Cells $\left(1 \times 10^{6}\right)$ were collected and subjected to fixation with $3 \%$ fresh glutaraldehyde and $1.5 \%$ paraformaldehyde solution at $4{ }^{\circ} \mathrm{C}$ for $1 \mathrm{~h}$, followed by post-fixation with $1 \%$ osmium tetroxide and $1.5 \%$ potassium ferrocyanide solution at $4^{\circ} \mathrm{C}$ for $1.5 \mathrm{~h}$. The cells were dehydrated with a graded series of ethanol solution and embedded in Epon-618. Ultrathin sections were cut to stain with uranyl acetate and lead citrate, and observed under TEM (Phillips, Madison, WI, USA).

qPCR detects gene expression. Total RNA was extracted using TRIzol reagent (Life Technologies, Rockville, MD, USA). The primer sequences of each gene are listed in Table I. The first-strand cDNA was generated using MMLV transcriptase [New England Biolabs (NEB), Ipswich, MA, USA], and qPCR was performed with FastStar Universal SYBR-Green Master (Roche, Foster City, CA, USA) in triplicate in an Applied Biosystems StepOnePlus Real-Time PCR system (Life Technologies). Endogenous mRNA values were normalized to the level of $\beta$-actin mRNA.

Western blot analysis. The cells were prepared by washing with ice-cold PBS and lysed. Protein concentrations were measured using the bicinchoninic acid (BCA) assay. Equal amounts of protein were loaded and separated by SDS-PAGE, and transferred to a nitrocellulose membrane. The membrane was blocked in 5\% milk in TBST [0.1\% Tween-20, $20 \mathrm{mM}$ Tris (pH 7.4) and $150 \mathrm{mM} \mathrm{NaCl}$ ] for $2 \mathrm{~h}$ at room temperature, followed by overnight incubation with the primary antibody at $4^{\circ} \mathrm{C}$. The membrane was washed and incubated with a horseradish peroxidase-conjugated secondary antibody for
Table I. Primer sequences.

\begin{tabular}{ll}
\hline Gene name & \multicolumn{1}{c}{ Primer sequence (5' to 3') } \\
\hline HBV X & F: ACTCTCTCGTCCCCTTCTCC \\
& R: GGTCGTTGACATTGCTGAGA \\
COXIII & F: CCCGCTAAATCCCCTA'AAG \\
& R: GGAAGCCTGTGGCT'CAAAA \\
$\beta$-actin & F: CTCCATCCTGGCCTCGCTGT \\
& R: GCTGTCACCTTCACCGTTCC
\end{tabular}

F, forward; R, reverse.

$1 \mathrm{~h}$ at room temperature. Proteins were visualized using an enhanced chemiluminescence (ECL) kit (ZSGB-BIO Co. Ltd., Beijing, China). Images of the blots were captured using the Image Scanner (Epson, Nagano, Japan). The band intensity was quantified using Image J software.

Statistical analysis. Each set of experiments was repeated at least three times with similar results. Results were presented as means \pm standard deviations (SD). Statistical evaluation was carried out by one-way analysis of variance (ANOVA). $\mathrm{P}<0.05$ was considered to indicate a statistically significant result.

\section{Results}

Construction and identification of an HL-7702 cell line stably transduced with a lentivirus expressing the HBx gene. Lentiviral vectors lead to prolonged transgene expression (24). In the present study, we used the vectors to establish longterm HBx expressing HL-7702 cells in vitro. The sequences of the recombinant vector PCMV.flag-HBx examined by the sequencing process showed that the full-length $H B x$ gene had been successfully subcloned into the lentiviral vector (data not shown). The titre of the recombinant lentivirus was $8.61 \times 10^{7} \mathrm{TU} / \mathrm{ml}$. GFP expression of HL-7702-HBx and HL-7702-mock were observed by fluorescence microscopy. The GFP-positive cells were 95-98\% (Fig. 1A). Expression of HBx mRNA and protein were detected in HL-7702-HBx cells (Fig. 1B and C). This showed that the HL-7702 cell line stably expressing HBx was established.

HBx co-localizes with inner mitochondrial protein COXIII in $H L-7702$ cells. Our recent studies have verified that COXIII interacts with $\mathrm{HBx}$ (11-13). Toconfirm this finding, we subjected HL-7702-HBx and control cells to fluorescent staining and examination by confocal microscopy. The co-localization of HBx protein (green color) with COXIII protein (blue color) in mitochondria (red color) was in white in HL-7702-HBx cells, while in HL-7702 cells the co-localization of COXIII protein (blue color) and mitochondria (red color) was in purple (Fig. 2). In addition, HBx localized in the nucleus and cytoplasm, with a fraction of cytosolic HBx co-localized with mitochondria as identified in previous studies (4-9). Thus, our study demonstrated that HBx co-localized with inner mitochondrial protein COXIII in HL-7702 cells. 
A

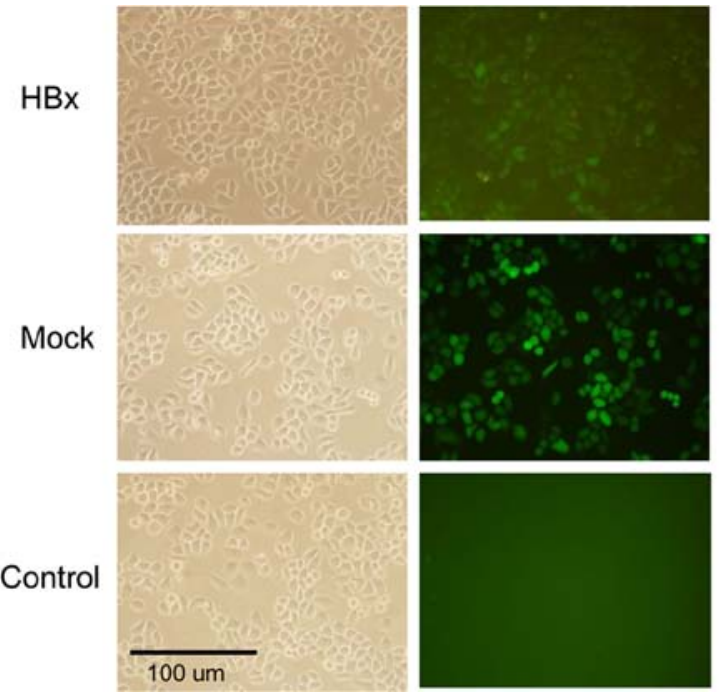

B

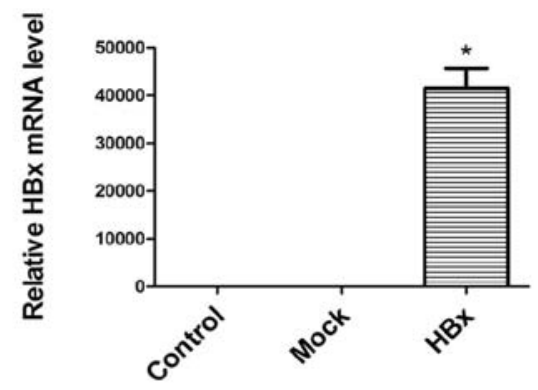

C

Control Mock HBx

Figure 1. Construction of an HL-7702 cell line stably expressing HBx gene. HBx, mock and control represented HL-7702-HBx, HL-7702-mock and HL-7702 cells, respectively. (A) Fluorescence microscope analysis of HL-7702 cells transduced with lentiviral vectors expressing green fluorescent protein (GFP). Cells were observed by visible light (left) and fluorescence microscope (right), respectively (original magnification, x200). (B) qPCR detection of HBx mRNA expression. (C) Western blot detection of HBx protein expression by the antibody specific against flag protein and $\beta$-actin.

A

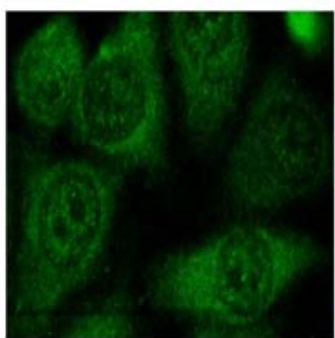

B

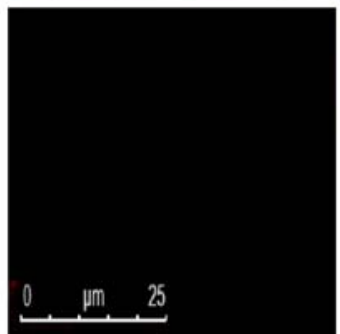

COXIII
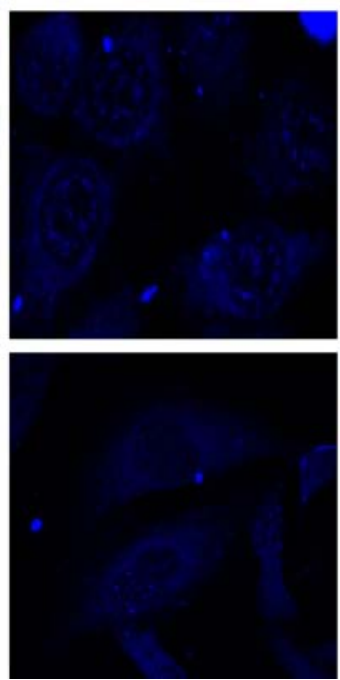

Mito Tracker
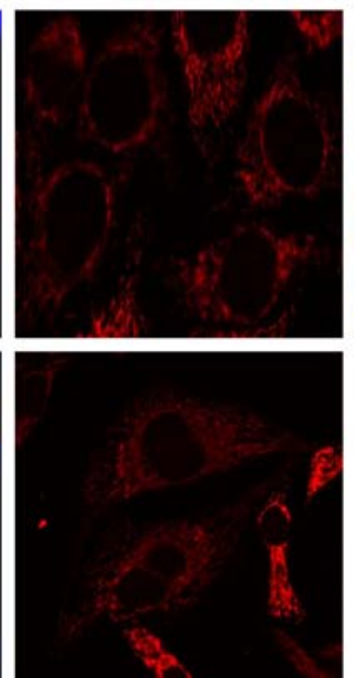

Merged
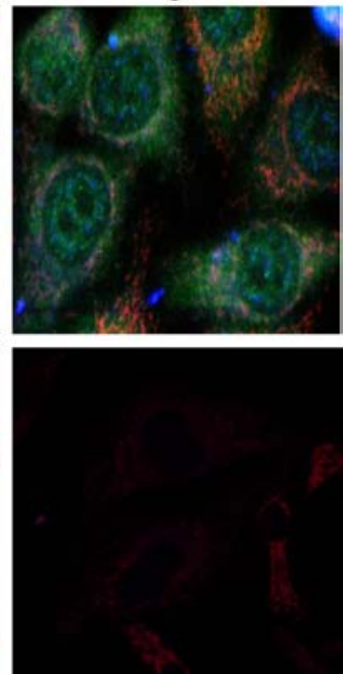

Figure 2. Co-localization of HBx with COXIII in mitochondria by confocal microscopy. Subcellular localization of HBx and COXIII were visualized by immunofluorescence staining with anti-flag antibody (green) and anti-COXIII (blue) antibodies. Mitochondria were stained with MitoTracker (red). The merged images are shown in the right panel. (A) HL-7702-HBx cells, (B) HL-7702 cells (original magnification, x800).

Upregulation of the mitochondrial function in $H L-7702-H B x$ cells. COXIII is an essential component of the mitochondrial respiratory chain. Thus, the co-localization of $\mathrm{HBx}$ and COXIII was expected to induce an alteration in the normal mitochondrial electron flow. To determine mitochondrial function alteration in HL-7702-HBx cells, we compared the expression of mitochondrial protein subunits of complexes IV (COXIII subunit). The expression of COXIII protein was increased in HL-7702-HBx cells, while the RNA level did not change (Fig. 3A and B). The result indicated that $\mathrm{HBx}$ regulated COXIII gene function at the post-transcription level. Then we measured the activities of COX (Fig. 3C). As expected, COX activity of HL-7702-HBx cells was significantly increased, correlating with their upregulated protein activities. However, there was no difference in the $\Delta \Psi \mathrm{m}$ of any of the cells (Fig. 3D).
Changes of mitochondrial ultrastructure in HBx steadily expressed cells. Abnormal aggregation of mitochondria has been found in HBx-expressing cells $(9,25)$. To assess the mitochondrial morphological consequences in HL-7702-HBx cells, we observed the mitochondria, nuclei and other intracellular membrane structures by TEM. As shown in Fig. 4, mitochondria had only slight swelling in HL-7702-HBx cells. Mitochondria in HL-7702-mock and HL-7702 cells were round or oval, and their membrane was integral, crest was dense and arranged in order. Our results indicated that cell and nuclear morphology were not significantly changed and there was no mitochondrial aggregation in any of the three groups.

Upregulation of ROS production in HL-7702-HBx cells. Since we demonstrated that $\mathrm{HBx}$ upregulates genes are involved 
A

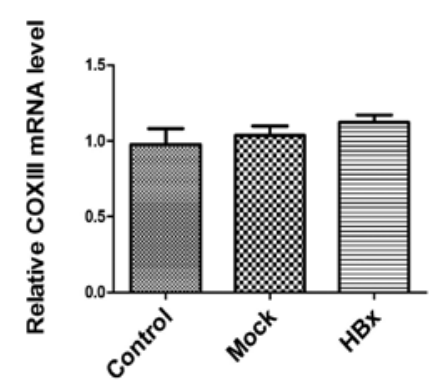

C

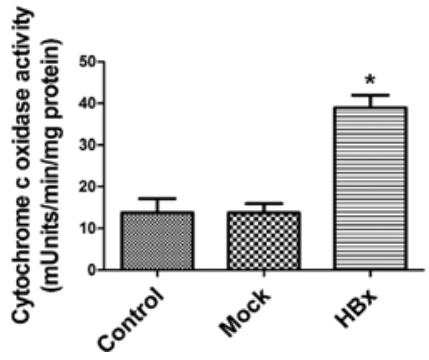

B

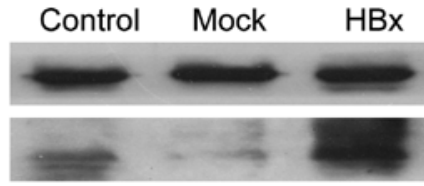

$\beta$-actin $43 \mathrm{kDa}$

COXIII $30 \mathrm{kDa}$

D
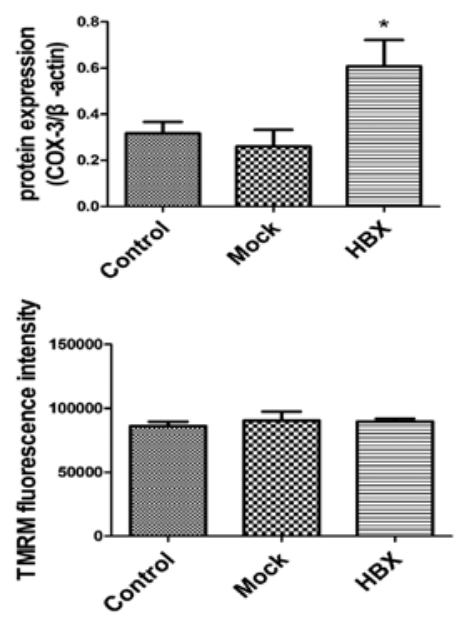

Figure 3. HBx leads to the upregulation of mitochondrial function in HL-7702-HBx cells. HBx, mock and control represented HL-7702-HBx, HL-7702-mock and HL-7702 cells, respectively. (A) Expression of COXIII mRNA was detected using qPCR. Levels of $\beta$-actin mRNA were used as an internal control. (B) Levels of COXIII protein were detected in lysates of HL-7702, HL-7702-mock and HL-7702-HBx cells using western blotting by antibody specific against COXIII protein and $\beta$-actin. (C) Changes of cytochrome $c$ oxidase (COX) activity were determinated using the Cytochrome $c$ Oxidase Assay kit. (D) Mitochondrial membrane potential was measured using fluorescence dye, TMRM, followed by flow cytometry. Values are the means \pm SD for at least three independent experiments. ${ }^{*} \mathrm{P}<0.05$.
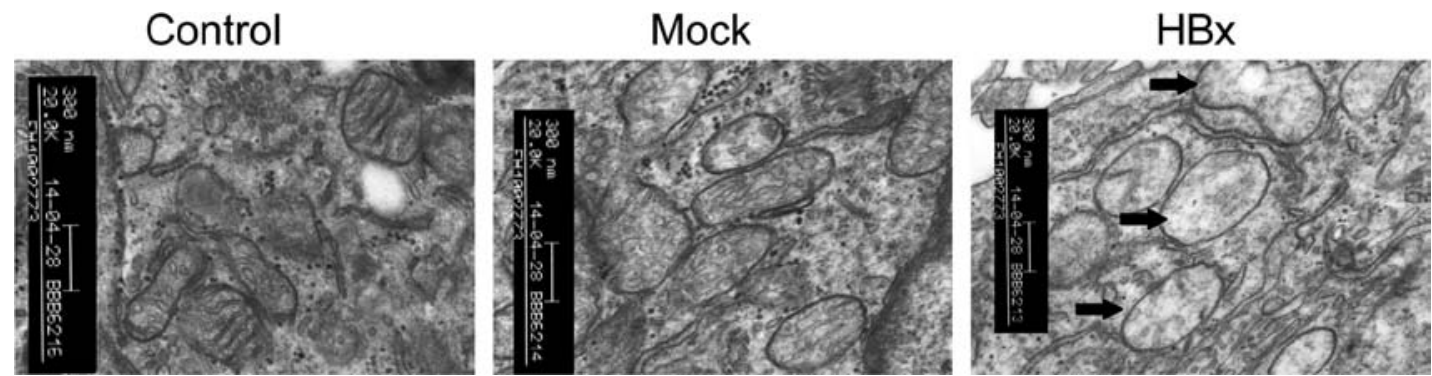

Figure 4. Changes of mitochondrial ultrastructure in HL-7702-HBx cells. Morphological changes of mitochondria were examined by electronic microscopy (original magnification, x16,000). Slight swelling in mitochondria was found in the HL-7702-HBx cells (shown by the arrow).
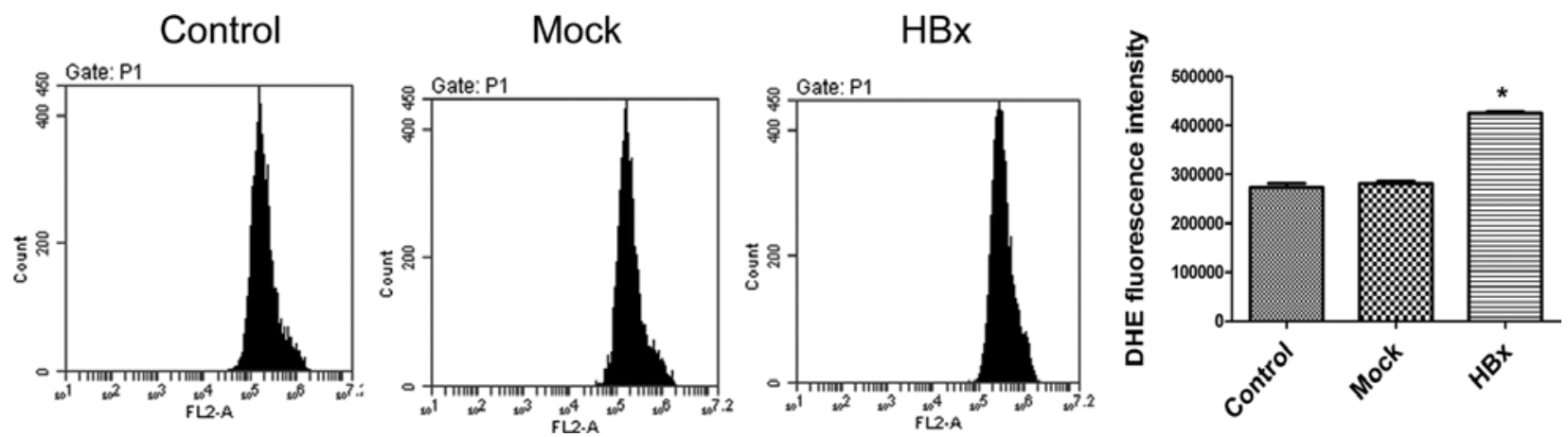

Figure 5. HBx induces ROS generation in HL-7702 cells. HBx, mock and control represented HL-7702-HBx, HL-7702-mock and HL-7702 cells, respectively. Intracellular ROS levels were determined using fluorescence dye, DHE, followed by flow cytometry. Values are the means \pm SD for at least three independent experiments. ${ }^{*} \mathrm{P}<0.05$ compared with HL-7702 and HL-7702-mock cells.

in oxidative phosphorylation at mitochondria, a major ROS generator, we examined whether HBx induces ROS production in HBx-transfected cells. Therefore, we examined
ROS levels by flow cytometry using the DHE probe. Fig. 5 shows that DHE fluorescence revealed an increase in the HL-7702-HBx cells. 


\section{Discussion}

HBx plays a key role in the development of HBV-associated HCC (3). Using a variety of methods such as liposome preparation, electrical transduction and adenovirus can deliver $\mathrm{HBx}$. However, lentiviral vectors are more suitable for long-term expression in non-dividing or infrequently dividing cells due to advantages of low toxicity and lack of immunogenicity $(26,27)$. In the present study, we successfully established HL-7702 cells that stably expressed HBx in vitro using lentiviral vectors. They served as a biologically relevant system for exploring the effect of HBx in human normal hepatocytes in physiology.

HBx protein is located in the cytoplasm and partly in the nucleus (4). Accumulating evidence has demonstrated that a fraction of cytosolic HBx co-localizes with the mitochondria in different cell lines (5-10) in addition to the outer mitochondrial membrane protein, VDAC3 $(8,10)$. HBx has been found to localize to mitochondria, leading to mtDNA damage, which encodes COXIII subunit $(18,28)$. Recent findings have shown that COXIII also interacts with HBx (11-13). These findings strongly predict the interaction between HBx and COXIII. However, the localization of HBx within the inner mitochondrial membrane and the effect of HBx on mitochondrial physiology in HL-7702 cells have not been previously addressed. To the best of our knowledge, our results were the first to suggest the inner mitochondrial membrane localization of HBx in HL-7702 cells (Fig. 2). These results provide insight into the mitochondrial localization of $\mathrm{HBx}$.

COX is the terminal complex of the electron transport chain and its biogenesis is a critical part of mitochondrial biogenesis (29,30). Mounting evidence suggests that the upregulation of COX has been found in human prostate (31) and breast carcinoma (32), and human colon cancers (22). In the present study, we characterized the upregulation of COX biogenesis in HL-7702-HBx cells, including the increasing protein expression of COXIII and upregulation of the activities of COX, which may provide more energy for cells to grow. These results were consistent with recent studies showing HBx (HBV serotype ayw) upregulated the protein expression of COXIII and activities of COX in HepG2 cells which stably expressed HBx using lentiviral vectors (13). By contrast, HBx (HBV genotype B) downregulated COXIII expression and inhibited COX activity in HL-7702 cells that expressed HBx using eukaryotic vector (33). The reasons for this discrepancy include that HBx plays pro-apoptotic and anti-apoptotic roles in different situations (34), and different genotypes of HBV may play different roles. On the other hand, HBx in long-term transfected cells is different from that in transiently transfected cells. COX accepts electrons from cytochrome $c$ and transfers them to molecular oxygen. At the same time protons are pumped across the inner mitochondrial membrane leading to the generation of $\Delta \Psi \mathrm{m}$ (35). It has been reported that $\mathrm{HBx}$ located in mitochondria may cause loss of $\Delta \Psi \mathrm{m}$ in hepatoma cells $(8,36)$. By contrast, in our system, the co-localization of $\mathrm{HBx}$ with COXIII protein did not induce changes in $\Delta \Psi \mathrm{m}$. We consider that long-term HBx-transfected cells in our system were more stable than the abovementioned $\mathrm{HBx}$ transiently transfected cells. Thus, the upregulation of COX biogenesis did not result in $\Delta \Psi \mathrm{m}$ alteration. Additionally, the role of HBx in human normal hepatocytes (HL-7702 cells) may be different from that in hepatoma cells. We then showed the slight swelling of mitochondria in HL-7702-HBx cells accompanied by increased respiratory enzyme activities and protein levels in mitochondria, as indicated by electron microscopy. This is an adaptive state for mitochondria, which adapt to the change of microenvironment. These results suggest that the co-localization of HBx with COXIII led to changes of mitochondrial function and morphology.

Mitochondria are the source of ROS (15). COX encoded gene expression changes are associated with the development of tumors mainly through an increase in ROS in mitochondria oxidative phosphorylation (37-40). In the present study, we observed that HBx expression elevated the generation of ROS (Fig. 5). HBx increases the level of mitochondrial ROS, which is associated with hepatocellular carcinogenesis $(15,41)$, although the mechanism involved remains controversial.

In summary, we have demonstrated that the HBx protein co-localizes with the inner mitochondrial membrane protein COXIII in HL-7702 cells, leading to changes of mitochondrial function, morphology and increasing the generation of mitochondrial ROS. These results provide insight into the molecular mechanism of HBV-associated HCC.

\section{Acknowledgements}

The present study was supported by the National Natural Science (grant no. 81300321) from the Foundation of China, the Key Clinical Specialty Discipline Construction Program of Fujian Province (2012-149), and Young and MiddleAged Personnel Training Project of Fujian Province Health Department (2014-ZQN-ZD-9). We thank Professor Bouchard for the PcDNA3.1-X plasmid.

\section{References}

1. Motavaf M, Safari S, Saffari Jourshari M and Alavian SM: Hepatitis B virus-induced hepatocellular carcinoma: The role of the virus x protein. Acta Virol 57: 389-396, 2013.

2. Neuveut C, Wei Y and Buendia MA: Mechanisms of HBV-related hepatocarcinogenesis. J Hepatol 52: 594-604, 2010.

3. Bouchard MJ and Schneider RJ: The enigmatic X gene of hepatitis B virus. J Virol 78: 12725-12734, 2004.

4. Henkler F, Hoare J, Waseem N, Goldin RD, McGarvey MJ, Koshy R and King IA: Intracellular localization of the hepatitis B virus HBx protein. J Gen Virol 82: 871-882, 2001.

5. Clippinger AJ and Bouchard MJ: Hepatitis B virus HBx protein localizes to mitochondria in primary rat hepatocytes and modulates mitochondrial membrane potential. J Virol 82: 6798-6811, 2008.

6. Li SK, Ho SF, Tsui KW, Fung KP and Waye MY: Identification of functionally important amino acid residues in the mitochondria targeting sequence of hepatitis B virus X protein. Virology 381: 81-88, 2008.

7. Chen J and Siddiqui A: Hepatitis B virus X protein stimulates the mitochondrial translocation of Raf-1 via oxidative stress. J Virol 81: 6757-6760, 2007.

8. Rahmani Z, Huh KW, Lasher R and Siddiqui A: Hepatitis B virus $X$ protein colocalizes to mitochondria with a human voltage-dependent anion channel, HVDAC 3 , and alters its transmembrane potential. J Virol 74: 2840-2846, 2000.

9. Takada S, Shirakata Y, Kaneniwa N and Koike K: Association of hepatitis B virus X protein with mitochondria causes mitochondrial aggregation at the nuclear periphery, leading to cell death. Oncogene 18: 6965-6973, 1999.

10. Huh KW and Siddiqui A: Characterization of the mitochondrial association of hepatitis B virus X protein, HBx. Mitochondrion 1: 349-359, 2002.

11. Li D, Wang XZ, Yu JP, Chen ZX, Huang YH and Tao QM: Cytochrome $c$ oxidase III interacts with hepatitis B virus X protein in vivo by yeast two-hybrid system. World J Gastroenterol 10: 2805-2808, 2004. 
12. Wang XZ, Li D, Tao QM, Lin N and Chen ZX: A novel hepatitis B virus $X$-interactive protein: Cytochrome $C$ oxidase III. J Gastroenterol Hepatol 21: 711-715, 2006.

13. Zheng BY, Fang XF, Zou LY, Huang YH, Chen ZX, Li D Zhou LY, Chen H and Wang XZ: The co-localization of HBx and COXIII upregulates COX-2 promoting HepG2 cell growth. Int J Oncol 45: 1143-1150, 2014.

14. Salvioli S, Bonafè M, Capri M, Monti D and Franceschi C: Mitochondria, aging and longevity - a new perspective. FEBS Lett 492: 9-13, 2001.

15. Lee YI, Hwang JM, Im JH, Lee YI, Kim NS, Kim DG, Yu DY, Moon HB and Park SK: Human hepatitis B virus-X protein alters mitochondrial function and physiology in human liver cells. J Biol Chem 279: 15460-15471, 2004.

16. Nishida N, Arizumi T, Takita M, et al: Reactive oxygen species induce epigenetic instability through the formation of 8-hydroxydeoxyguanosine in human hepatocarcinogenesis. Dig Dis 31: 459-466, 2013.

17. Castello G, Costantini S and Scala S: Targeting the inflammation in HCV-associated hepatocellular carcinoma: A role in the prevention and treatment. J Transl Med 8: 109, 2010.

18. Mkaouar-Rebai E, Ellouze E, Chamkha I, Kammoun F, Triki C and Fakhfakh F: Molecular-clinical correlation in a family with a novel heteroplasmic Leigh syndrome missense mutation in the mitochondrial cytochrome $c$ oxidase III gene. J Child Neurol 26 12-20, 2011.

19. Bauerfeld CP, Rastogi R, Pirockinaite G, Lee I, Hüttemann M, Monks B, Birnbaum MJ, Franchi L, Nuñez G and Samavati L: TLR4-mediated AKT activation is MyD88/TRIF dependent and critical for induction of oxidative phosphorylation and mitochondrial transcription factor A in murine macrophages. J Immunol 188: 2847-2857, 2012.

20. Liang L, Qu L and Ding Y: Protein and mRNA characterization in human colorectal carcinoma cell lines with different metastatic potentials. Cancer Invest 25: 427-434, 2007.

21. Bafna S, Singh AP, Moniaux N, Eudy JD, Meza JL and Batra SK: MUC4, a multifunctional transmembrane glycoprotein, induces oncogenic transformation of NIH3T3 mouse fibroblast cells. Cancer Res 68: 9231-9238, 2008

22. Wu H, Rao GN, Dai B and Singh P: Autocrine gastrins in colon cancer cells up-regulate cytochrome $c$ oxidase $\mathrm{Vb}$ and downregulate efflux of cytochrome $c$ and activation of caspase-3. J Biol Chem 275: 32491-32498, 2000.

23. Nybo K: GFP imaging in fixed cells. Biotechniques 52: 359-360, 2012.

24. Hu B, Tai A and Wang P: Immunization delivered by lentiviral vectors for cancer and infectious diseases. Immunol Rev 239: 45-61, 2011.

25. Kim S, Kim HY, Lee S, Kim SW, Sohn S, Kim K and Cho H: Hepatitis $\mathrm{B}$ virus $\mathrm{x}$ protein induces perinuclear mitochondrial clustering in microtubule- and Dynein-dependent manners. J Virol 81: 1714-1726, 2007.

26. Naldini L, Blömer U, Gallay P, Ory D, Mulligan R, Gage FH, Verma IM and Trono D: In vivo gene delivery and stable transduction of nondividing cells by a lentiviral vector. Science 272 263-267, 1996.
27. Ohashi K, Park F, Schwall R and Kay M: Efficient gene transduction to cultured hepatocytes by HIV-1 derived lentiviral vector. Transplant Proc 34: 1431-1433, 2002.

28. Jung SY and Kim YJ: C-terminal region of HBx is crucial for mitochondrial DNA damage. Cancer Lett 331: 76-83, 2013.

29. Zaslavsky D and Gennis RB: Proton pumping by cytochrome oxidase: Progress, problems and postulates. Biochim Biophys Acta 1458: 164-179, 2000.

30. Qi Z, He J, Su Y, et al: Physical exercise regulates p53 activity targeting $\mathrm{SCO} 2$ and increases mitochondrial COX biogenesis in cardiac muscle with age. PLoS One 6: e21140,2011.

31. Wang FL, Wang Y, Wong WK, Liu Y, Addivinola FJ, Liang P, Chen LB, Kantoff PW and Pardee AB: Two differentially expressed genes in normal human prostate tissue and in carcinoma. Cancer Res 56: 3634-3637, 1996.

32. Bini L, Magi B, Marzocchi B, et al: Protein expression profiles in human breast ductal carcinoma and histologically normal tissue. Electrophoresis 18: 2832-2841, 1997.

33. Lin N, Li D, Chen HY, Chen ZX and Wang XZ: [Effect of HBV X gene on mitochondria in HL-7702 cells]. Xi Bao Yu Fen Zi Mian Yi Xue Za Zhi 24: 972-974, 2008 (In Chinese).

34. Lee WP, Lan KH, Li CP, Chao Y, Lin HC and Lee SD: Pro-apoptotic or anti-apoptotic property of $\mathrm{X}$ protein of hepatitis B virus is determined by phosphorylation at Ser31 by Akt. Arch Biochem Biophys 528: 156-162, 2012.

35. Adrie C, Bachelet M, Vayssier-Taussat M, Russo-Marie F, Bouchaert I, Adib-Conquy M, Cavaillon JM, Pinsky MR, Dhainaut JF and Polla BS: Mitochondrial membrane potential and apoptosis peripheral blood monocytes in severe human sepsis. Am J Respir Crit Care Med 164: 389-395, 2001.

36. Shirakata Y and Koike K: Hepatitis B virus X protein induces cell death by causing loss of mitochondrial membrane potential. J Biol Chem 278: 22071-22078, 2003.

37. Ray AM, Zuhlke KA, Levin AM, Douglas JA, Cooney KA and Petros JA: Sequence variation in the mitochondrial gene cytochrome $c$ oxidase subunit I and prostate cancer in African American men. Prostate 69: 956-960, 2009.

38. Athar M, Chaudhury NK, Hussain ME and Varshney R: Hoechst 33342 induced reactive oxygen species and impaired expression of cytochrome $c$ oxidase subunit 1 leading to cell death in irradiated human cancer cells. Mol Cell Biochem 352: 281-292, 2011.

39. Bernstein C, Facista A, Nguyen H, et al: Cancer and age related colonic crypt deficiencies in cytochrome $c$ oxidase I. World $\mathrm{J}$ Gastrointest Oncol 2: 429-442, 2010

40. Gutierrez-Gonzalez L, Graham TA, Rodriguez-Justo M, et al: The clonal origins of dysplasia from intestinal metaplasia in the human stomach. Gastroenterology 140: 1251-1260.e1-6, 2011.

41. Ha HL and Yu DY: HBx-induced reactive oxygen species activates hepatocellular carcinogenesis via dysregulation of PTEN/Akt pathway. World J Gastroenterol 16: 4932-4937, 2010. 